\title{
Parecoxib nel trattamento del dolore post-operatorio: considerazioni farmacoeconomiche
}

Federica Mathis*, Lorenzo Pradelli§, Mario Eandi§

\section{ABSTRACT}

Parecoxib sodium is the first selective COX- 2 inhibitor to be available for parenteral administration. This pharmaceutical feature, alongside its favorable efficacy and safety profile, makes the new drug particularly suitable for simple and effective management of post-surgical pain.

Aim of this work is to describe the pharmacoeconomical profile of the drug in the Italian setting, focusing on the comparison with parenteral ketorolac, the most widely prescribed treatment for post-surgical pain in Italy.

The first part of the article describes the most important pharmacological characteristics of parecoxib and reviews the main efficacy and safety studies that were performed on patients presenting with post-surgical pain.

A similar article about parecoxib was published on "Farmeconomia e percorsi terapeutici" 2003; 4(1): 39-50. At that moment, the price to the public wasn't yet fixed and therefore the pharmacoeconomical analysis was based on estimated price. Moreover, during this year, extra studies were published on the efficacy of parecoxib on patients with post-surgical pain.

For these reasons, we intend to present here an updated version of the profile.

Farmeconomia e percorsi terapeutici 2004; 5 (4): 227-242

Un profilo farmacoeconomico del parecoxib sodico era già stato pubblicato sul primo numero del 2003 di questa rivista. Al momento della pubblicazione, il prezzo al pubblico del prodotto a base di parecoxib non era ancora stato fissato, per cui le considerazioni economiche si basavano su un prezzo stimato. Nel corso di questo anno sono stati pubblicati ulteriori studi per la valutazione della molecola nel trattamento del dolore post-operatorio ed è stato stabilito il prezzo al pubblico: per questi motivi abbiamo ritenuto utile pubblicare una versione aggiornata del testo.

\section{INTRODUZIONE}

Le caratteristiche richieste ad un farmaco per il trattamento parenterale del dolore postoperatorio sono: una rapida azione analgesica, una efficacia protratta fino a garantire una adeguata copertura analgesica, nonché un profilo di sicurezza e di tollerabilità che permetta di evitare complicanze iatrogene in pazienti già a rischio di sviluppare eventi avversi tipici della fase post-chirurgica.

Farmaci ampiamente usati nella terapia del dolore acuto, in particolare post-chirurgico, sono gli oppiacei e i farmaci antinfiammatori non steroidei (FANS), che, se da un lato sono dotati di un elevato potere analgesico, dall' altro mostrano una elevata incidenza e una ampia varietà di effetti collaterali: depressione respiratoria, sedazione, nausea, vomito, ritenzione urinaria, stipsi e riduzione della motilità gastroenterica fino all'ileo paralitico per quanto riguarda gli oppiacei, ulcere ed emorragie gastrointestinali, danni renali ed epatici, nonché un'inibizione della funzionalità piastrinica con possibile aumento dei sanguinamenti post-chirurgici per quanto riguarda i FANS.

La scoperta e la caratterizzazione dei diversi isoenzimi della cicloossigenasi (COX), nonché la comprensione delle principali attività svolte rispettivamente dalla COX-1 e dalla COX-2 nell'organismo, ha permesso di sintetizzare FANS in grado di inibire selettivamente la COX-2. Parecoxib sodico è il primo inibitore selettivo della COX-2 disponibile in formulazione iniettabile, per via sia endovenosa sia intramuscolare.

\author{
* Osservatorio \\ Epidemiologico \\ delle Dipendenze \\ della Regione Piemonte \\ § Farmacologia clinica \\ Università di Torino
}


Con questo lavoro si intende presentare un profilo farmacologico clinico e farmacoeconomico del parecoxib iniettabile.

\section{Caratteristiche farmacologiche del parecoxib}

Parecoxib è il sale di sodio della N-[4-(5metil-3-fenilisossazol-4-il)fenilsulfonil]propionamide. È un profarmaco inattivo che, dopo somministrazione parenterale, viene rapidamente idrolizzato in valdecoxib, molecola lipofila farmacologicamente attiva, a cui è interamente ascrivibile l' azione del farmaco.

Valdecoxib inibisce selettivamente la cicloossigenasi di tipo 2, grazie al suo legame irreversibile al sito attivo dell'enzima, cui conseguono delle modificazioni conformazionali della COX-2 a livello del sito catalitico e della tasca laterale.

La selettività di valdecoxib è molto pronunciata: in studi in vitro, una concentrazione di $0,005 \mathrm{~mol} / \mathrm{L}$ è sufficiente per ridurre del $50 \%$ l'attività della COX-2, mentre per ottenere la stessa riduzione percentuale dell' attività della COX-1 la concentrazione richiesta è di $140 \mathrm{~mol} /$ L, molto superiore ai livelli ottenuti con dosi terapeutiche di parecoxib. Test analoghi condotti con coxib ex vivo hanno evidenziato rapporti di selettività inferiori ma pur sempre elevati [1].

Il legame selettivo del valdecoxib con la COX-2 permette di non interferire con l'isoenzima COX-1, che, costitutivamente espresso nella maggior parte delle cellule, svolge una funzione sostanzialmente citoprotettiva (per esempio a livello dell'apparato gastro-intestinale) e regolatoria della aggregazione piastrinica e della funzionalità renale [2].

La COX-2, in larga misura inattiva nei tessuti sani ma indotta in seguito a stimoli flogistici e proliferativi, risulta invece essere l'isoforma principalmente responsabile della produzione delle prostaglandine coinvolte nell'infiammazione, nel dolore e nella febbre [2,3].

Come dimostrato in modelli sperimentali, infatti, all'induzione di una infiammazione periferica o di un danno tissutale, mentre non si riscontrano modificazioni riguardanti l'RNAm della COX-1, si assiste ad un aumento notevole dell' espressione inducibile della COX-2, sia nella sede periferica lesa, sia a livello spinale ed in altre regioni del SNC quali il ponte, il mesencefalo, l'ipotalamo e il talamo. In parallelo si ha un incremento delle prostaglandine (principalmente PGE2) sia nel sito della lesione, dove determinano una sensibilizzazione dei nocicettori, sia a livello del midollo spinale, dove incrementano ulteriormente la sensibilità al dolore, con fenomeni di iperalgesia e allodinia $[2,3]$. L'inibizione selettiva della COX-2 riduce i livelli della prostaglandina $\mathrm{E}_{2}$ nel CSF e la conseguente iperalgesia. Secondo gli studi condotti da Samad e coll. [3], inoltre, la COX-2 è espressa in quantitativi rilevanti nel midollo spinale anche in presenza di un blocco sensoriale al sito dell'infiammazione periferica. Tale induzione centrale della COX-2 potrebbe essere causata da un segnale bioumorale inviato dal sito dell' infiammazione attraverso la barriera ematoencefalica. Da ciò si può dedurre che, al fine di ottenere una analgesia ottimale, gli inibitori selettivi della COX-2 devono essere in grado di agire sia a livello centrale, sia a livello periferico.

Grazie a spiccate caratteristiche di lipofilia, il valdecoxib è in grado di attraversare rapidamente la barriera ematoencefalica, con conseguente inibizione delle COX-2 anche a livello midollare.

\section{FARMACOCINETICA}

Le caratteristiche farmacocinetiche salienti del parecoxib sono riassunte in tabella 1 .

L'esposizione sistemica a valdecoxib risulta indipendente dalla via di somministrazione di parecoxib, tuttavia la Cmax di parecoxib dopo somministrazione intramuscolare appare significativamente inferiore rispetto a quella osservata dopo somministrazione endovenosa, probabilmente a causa di un assorbimento extravascolare più lento dopo somministrazione intramuscolare. Data l'equivalenza nell'esposizione complessiva a valdecoxib dopo somministrazione endovenosa o intramuscolare, questo fenomeno non assume tuttavia caratteristiche rilevanti dal punto di vista clinico [4].

Un dimezzamento del dosaggio è richiesto in caso di peso corporeo inferiore a 50 chilogrammi o in presenza di insufficienza epatica di grado moderato. Non è necessaria una riduzione della posologia invece in caso di insufficienza renale o pazienti anziani. A causa della mancanza di studi specifici, non è raccomandato l'uso di parecoxib nei pazienti con grave alterazione della funzionalità epatica [5].

\section{STUDI CLINICI DI EFFICACIA}

L'efficacia di parecoxib nel trattamento a breve termine del dolore post-operatorio è stata esaminata in studi randomizzati, in doppio cieco, sia rispetto al placebo, sia rispetto alla terapia parenterale con i farmaci più utilizzati nella pratica clinica: ketorolac e morfina. I parametri utilizzati per valutare l'efficacia della 


\begin{tabular}{|c|c|c|c|c|c|c|}
\hline & $\begin{array}{l}\text { Cmax } \\
\text { (valdecoxib) }\end{array}$ & $\begin{array}{l}\text { Tmax } \\
\text { (valdecoxib) }\end{array}$ & Metabolismo & Metaboliti attivi & $\begin{array}{l}\text { Escrezione } \\
\text { (valdecoxib) }\end{array}$ & $\begin{array}{l}\text { Emivita } \\
\text { (valdecoxib) }\end{array}$ \\
\hline $\mathrm{IM}$ & $\begin{array}{l}0,5 \mu \mathrm{g} / \mathrm{ml} \\
(40 \mathrm{mg})\end{array}$ & $1,5-2,5$ ore & $\begin{array}{l}\text { Idrolizzato a } \\
\text { valdecoxib, poi } \\
\text { CYP3A4 e } \\
\text { CYP2C9 }\end{array}$ & $\begin{array}{l}\text { Valdecoxib e } \\
\text { SC-66905 }\end{array}$ & $\begin{array}{l}\text { Urinaria: meno } \\
\text { del } 5 \% \\
\text { immodificato }\end{array}$ & $7-10$ ore \\
\hline EV & $\begin{array}{l}0,5 \mu \mathrm{g} / \mathrm{ml} \\
(20 \mathrm{mg})\end{array}$ & 0,5 - 1 ora & & & & $\simeq 8$ ore \\
\hline
\end{tabular}

terapia sono: il sollievo dal dolore (PR), la differenza di intensità del dolore (PID), il tempo di insorgenza dell'effetto antalgico, l'intervallo di tempo precedente il ricorso a un trattamento analgesico aggiuntivo (tempo di rimedicazione), la valutazione globale da parte del paziente e da parte del medico dell'efficacia del trattamento, il risparmio nell'uso degli oppiacei.

\section{Trattamento del dolore post-operatorio dentale}

Lo studio di Mehlisch et al [6] ha confrontato l'efficacia della somministrazione endovenosa di dosi singole di parecoxib da $1,2,5$, $10,20,50$ o $100 \mathrm{mg}$ verso quella di una dose singola di ketorolac $30 \mathrm{mg}$ e di placebo, tutti somministrati entro sei ore dall'intervento chirurgico di estrazione di due o più terzi molari inclusi con osteotomia. La popolazione in studio era costituita da 454 pazienti di età compresa tra i 22 e i 25 anni, suddivisi in gruppi di 51 soggetti per ciascun dosaggio di parecoxib, 50 soggetti per il ketorolac e il placebo. L'intensità del dolore in condizioni basali risultava simile, da moderato a severo, nei diversi gruppi. Tranne che per i dosaggi di 1 o $2 \mathrm{mg}$, parecoxib sodico ha dimostrato una più rapida insorgenza dell' effetto antalgico (9-11 minuti contro 12 minuti per ketorolac, differenza non statisticamente significativa, e più di 24 ore per il placebo, differenza statisticamente significativa); una riduzione significativa dell'intensità del dolore (PID e PR) rispetto al placebo; per i dosaggi 20, 50 e $100 \mathrm{mg}$ una efficacia paragonabile al ketorolac nel ridurre l'intensità del dolore nelle prime sette ore successive alla somministrazione, ma una efficacia analgesica significativamente più prolungata nel tempo; un tempo di rimedicazione significativamente superiore nei dosaggi a 50 e 100 $\mathrm{mg}$, paragonabile a quello del ketorolac $30 \mathrm{mg}$ alla dose di parecoxib di $20 \mathrm{mg}$; un nettamente inferiore ricorso ad un trattamento aggiuntivo (54,9\% per parecoxib $100 \mathrm{mg}, 56,9 \%$ per il dosaggio a $50 \mathrm{mg}$, contro il $95.8 \%$ dei pazienti trattati con ketorolac). Il giudizio globale dei pazienti trattati con parecoxib è risultato migliore proporzionalmente all' aumentare della dose, ma anche per i dosaggi di 50 e 100 mg, paragonabile a quello dei pazienti trattati con ketorolac. Il giudizio è stato comunque significativamente migliore di quello espresso dai pazienti che hanno ricevuto placebo.

Risultati sovrapponibili emergono dallo studio di Daniels et al [7], che ha confrontato l'efficacia analgesica del parecoxib somministrato in dosi da 20 o $40 \mathrm{mg}$ (per via intramuscolare ed endovenosa) versus ketorolac $60 \mathrm{mg}$ e versus placebo in 304 pazienti sottoposti ad avulsione del terzo molare incluso. Per quanto riguarda il giudizio espresso dai pazienti sulla bontà del trattamento antalgico ricevuto, le percentuali di pazienti soddisfatti risultano simili nei gruppi trattati con parecoxib $40 \mathrm{mg}$ e ketorolac $60 \mathrm{mg}$ (80\% per entrambi i prodotti per via i.m.), mentre risultano leggermente inferiori nei gruppi che hanno ricevuto parecoxib $20 \mathrm{mg}$ (tabella 2).

I risultati di efficacia del parecoxib non è risultata influenzata dalla via di somministrazione, endovenosa o intramuscolare, anche se il tempo di rimedicazione è apparso più prolungato in seguito alla somministrazione intramuscolare.

In un altro studio clinico [8], non pubblicato, l'efficacia del parecoxib in singola dose di $20 \mathrm{mg}$, somministrata per via intramuscolare e endovenosa nel trattamento del dolore postoperatorio dentale è stata confrontata con una serie di competitors: valdecoxib $20 \mathrm{mg}$ per os, tramadolo $100 \mathrm{mg}$ per os, ibuprofene 400 mg per os e placebo.

In tale studio, la durata dell'effetto analgesico di parecoxib e valdecoxib è risultata superiore a quella ottenuta con ketorolac e ibuprofene e l'insorgenza di tale effetto è risultata significativamente più rapida con parecoxib che con ibuprofene.

\section{Trattamento del dolore post-operatorio ortopedico}

Nello studio di Hubbard et al [9] è stata valutata l'efficacia analgesica di dosi multiple di parecoxib sodico rispetto al placebo $(n=63)$, in 195 pazienti sottoposti a intervento di artroprotesi totale del ginocchio. La somministrazione endovenosa di $20 \mathrm{mg}(\mathrm{n}=65)$
Tabella 1 Parametri farmacocinetici del parecoxib/valdecoxib 


\begin{tabular}{|c|c|c|c|c|}
\hline Gruppo di trattamento & $\begin{array}{l}\text { Nr. di pazienti che } \\
\text { hanno percepito } \\
\text { l'effetto antalgico }\end{array}$ & $\begin{array}{l}\text { Tempo medio di } \\
\text { insorgenza dell'effetto } \\
\text { antalgico (ore:min) }\end{array}$ & $\begin{array}{l}\% \text { di pazienti che } \\
\text { esprimono giudizio } \\
\text { buono o eccellente }\end{array}$ & $\begin{array}{l}\% \text { di pazienti che } \\
\text { richiedono rescue } \\
\text { medication }\end{array}$ \\
\hline \multicolumn{5}{|l|}{ Parecoxib } \\
\hline $\begin{array}{l}20 \mathrm{mg} I \mathrm{M} \\
(\mathrm{n}=51)\end{array}$ & $38(75 \%)$ & $00: 13$ & $66 \%$ & $76 \%$ \\
\hline $\begin{array}{l}20 \text { mg EV } \\
(n=50)\end{array}$ & 39 (78\%) & $00: 13$ & $70 \%$ & $80 \%$ \\
\hline $\begin{array}{l}40 \mathrm{mg} I M \\
(\mathrm{n}=50)\end{array}$ & 47 (94\%) & $00: 13$ & $80 \%$ & $50 \%$ \\
\hline $\begin{array}{l}40 \text { mg EV } \\
(n=51)\end{array}$ & 45 (88\%) & $00: 13$ & $82 \%$ & $53 \%$ \\
\hline \multicolumn{5}{|l|}{ Ketorolac } \\
\hline $\begin{array}{l}60 \mathrm{mg} \mathrm{IM} \\
(\mathrm{n}=51)\end{array}$ & $41(80 \%)$ & $00: 12$ & $80 \%$ & $84 \%$ \\
\hline \multicolumn{5}{|l|}{ Placebo } \\
\hline$(n=51)$ & 9 (18\%) & $>24: 00$ & & $94 \%$ \\
\hline
\end{tabular}

Tabella 2

Effetto antalgico: numero e percentuale di pazienti che lo hanno percepito, tempo medio di insorgenza, soddisfazione nei vari gruppi di trattamento e tempo medio di rescue medication [7] o $40 \mathrm{mg}(\mathrm{n}=67)$ di parecoxib è avvenuta al termine dell'operazione chirurgica e di seguito dopo 12,24 e 36 ore dalla somministrazione della prima dose. I pazienti avevano a disposizione dosi di morfina (1-2 mg) da assumere come patient-controlled analgesia (PCA) o in bolo. I pazienti trattati con parecoxib hanno dimostrato, indipendentemente dal dosaggio, sia una ridotta intensità del dolore, sia un significativo minor ricorso alla morfina, in particolare nelle prime ventiquattro ore (ricorso a morfina $15,6 \%$ e 27,8\% minore rispettivamente per dosi di 20 e $40 \mathrm{mg}$ di parecoxib versus placebo). Il loro giudizio complessivo inoltre è stato a favore del parecoxib.

Questi risultati sono confermati dallo studio di Malan et al [10], in cui, analogamente allo studio di Hubbard et al, la somministrazione di dosi multiple di parecoxib $20 \mathrm{mg}(\mathrm{n}=67)$ e di parecoxib $40 \mathrm{mg}(\mathrm{n}=64)$ è stata confrontata versus placebo $(n=70)$ in pazienti dotati di dosi di morfina da assumere come patient-controlled analgesia (PCA). I pazienti, sottoposti ad intervento di artroprotesi d'anca, avevano assunto un primo bolo di $4 \mathrm{mg}$ di morfina in concomitanza con la prima dose del farmaco loro assegnato. Quest'ultimo è stato loro nuovamente somministrato 12 e 24 ore dopo la prima dose. I risultati ottenuti sono analoghi a quelli descritti nello studio precedente.

Lo studio di Rasmussen et al [11] ha invece confrontato l'efficacia di una singola dose endovenosa di parecoxib $20 \mathrm{mg}(\mathrm{n}=43)$ o $40 \mathrm{mg}$ $(\mathrm{n}=42)$ versus morfina $4 \mathrm{mg}(\mathrm{n}=42)$, ketorolac $30 \mathrm{mg}(\mathrm{n}=42)$ o placebo $(\mathrm{n}=39)$, somministrata entro 6 ore dall'interruzione della terapia con morfina come PCA nella prima giornata successiva ad un intervento di artroprotesi mono- laterale totale di ginocchio. Il tempo di insorgenza dell' analgesia è risultato simile nei pazienti che avevano ricevuto una dose di $40 \mathrm{mg}$ di parecoxib (11 minuti) o $4 \mathrm{mg}$ di morfina (12 minuti) o $30 \mathrm{mg}$ di ketorolac (15 minuti). Analoga è stata inoltre la riduzione del dolore (PID) ottenuta con $40 \mathrm{mg}$ di parecoxib e $30 \mathrm{mg}$ di ketorolac, mentre la riduzione dell' intensità del dolore rispetto al basale è risultata significativamente superiore nei pazienti trattati con 40 $\mathrm{mg}$ di parecoxib rispetto a coloro che avevano ricevuto $4 \mathrm{mg}$ di morfina. Il giudizio sul trattamento ricevuto è stato significativamente migliore per i pazienti dei gruppi parecoxib $40 \mathrm{mg}$ e ketorolac rispetto a quello dei gruppi parecoxib $20 \mathrm{mg}$, morfina e placebo (percentuali di giudizio buono/eccellente rispettivamente del 79\%, 68\%, 51\%, 44\% e 38\%).

L'efficacia del parecoxib nel trattamento del dolore conseguente ad interventi di chirurgia ortopedica è stata valutata inoltre nel corso di due studi clinici $[12,13]$, facenti parte del protocollo di sviluppo clinico del farmaco ma non pubblicati, controllati verso placebo, ketorolac e morfina.

Il primo di questi studi ha valutato il parecoxib nel trattamento di pazienti sottoposti ad artroplastica del ginocchio che presentavano dolore di grado moderato-severo. Lo studio prevedeva la somministrazione di una singola dose di parecoxib $20 \mathrm{mg}$ EV, parecoxib $40 \mathrm{mg}$ EV, ketorolac $60 \mathrm{mg} \mathrm{EV}$, morfina $4 \mathrm{mg}$ EV o placebo sei ore dopo l'interruzione della terapia antalgica precoce con morfina somministrata tramite PCA. Il tempo necessario a raggiungere il picco di efficacia antidolorifica è stato valutato in aggiunta ai parametri considerati negli studi precedenti. Il tempo medio 
d'insorgenza dell' effetto antalgico è risultato di 11, 12 e 15 minuti per i pazienti trattati rispettivamente con parecoxib $40 \mathrm{mg}$, ketorolac e morfina. Il sollievo ottenuto con parecoxib 40 $\mathrm{mg}$ è stato significativamente superiore rispetto a quello riscontrato nei pazienti che avevano ricevuto $20 \mathrm{mg}$ di parecoxib (sollievo riscontrato rispettivamente nell' $85 \%$ e nel $65 \%$ dei trattati). I valori medi di PR ottenuti con parecoxib $40 \mathrm{mg}$ sono risultati superiori a quelli ottenuti con parecoxib $20 \mathrm{mg}$, morfina e placebo per tutte le 24 ore di osservazione (figura 1).

Ketorolac e parecoxib $40 \mathrm{mg}$ sono risultati paragonabili in termini di PR, tempo di rimedicazione, percentuali di pazienti che hanno richiesto la rimedicazione e che hanno espresso un giudizio positivo riguardo al trattamento ricevuto, mentre il picco di PR è risultato più precoce con parecoxib (1 ora) che con ketorolac ( 2 ore).

Anche nel secondo studio clinico, condotto su pazienti sottoposti a interventi di sostituzione dell'anca, parecoxib ha mostrato di essere paragonabile a ketorolac e superiore a morfina per quanto riguarda la rapidità d'insorgenza e la durata dell'effetto antalgico.

\section{Trattamento del dolore postoperatorio ginecologico}

Nello studio di Barton et al [14], l'effetto analgesico ottenuto con la somministrazione in una unica dose di 20 o $40 \mathrm{mg}$ di parecoxib, è stata confrontata con quella conseguente alla somministrazione di ketorolac $30 \mathrm{mg}$ o morfina $4 \mathrm{mg}$ o placebo. Il campione in studio era costituito da 202 donne che, nella giornata successiva all'intervento di isterectomia o miomectomia, avevano riferito un dolore moderato/severo (>45 mm sulla scala analogicovisiva, VAS). Per tutti i parametri di efficacia valutati, i risultati sono apparsi migliori nelle donne trattate con parecoxib e ketorolac rispetto a quelli ottenuti con morfina o placebo. L'efficacia del parecoxib (entrambi i dosaggi) risulta paragonabile a quella del ketorolac per quanto riguarda sia la rapidità di comparsa dell'analgesia, sia l'entità complessiva dell'analgesia raggiunta, sia il tempo di rimedicazione, mentre la durata dell' attività analgesica risulta superiore per parecoxib a $40 \mathrm{mg}$.

Risultati sovrapponibili sono stati ottenuti nello studio di Bikhazi et al [4], in cui è stata valutata l'efficacia sia di una singola dose, sia di un regime a dosi multiple. A 6 ore dall'interruzione della PCA, 208 pazienti sottoposte ad intervento di isterectomia o miomectomia sono

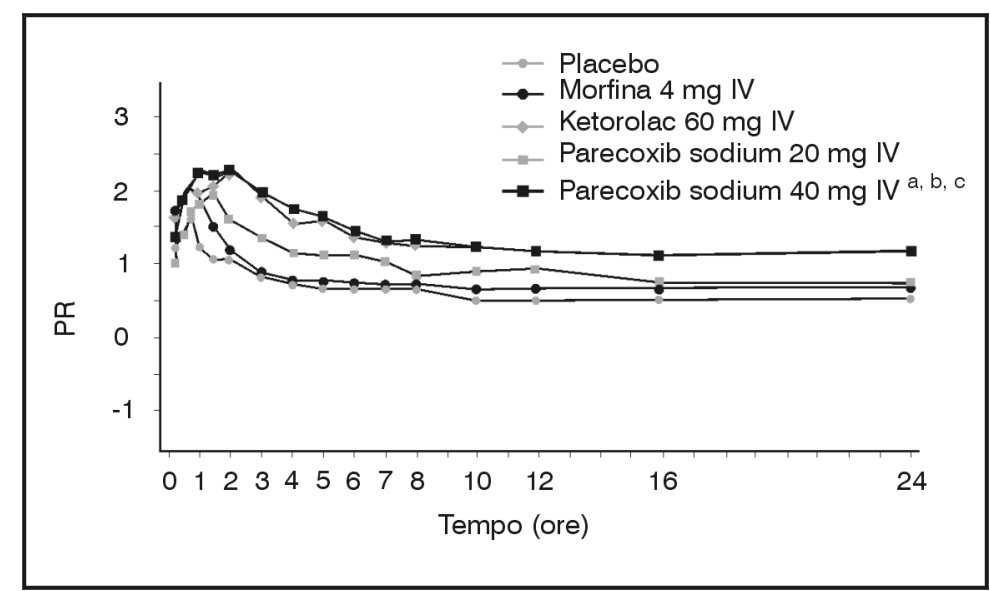

Figura 1

Valori medi di PR ai diversi intervalli di rilevazione

a $p<0,05$ vs morfina nell'intervallo $1,5-24$ ore

${ }^{b} p<0,05$ vs parecoxib sodico $20 \mathrm{mg}$ a 0,5/2-5/ 8/ 16/ 24 ore

c $p<0,05$ vs placebo a 0,5 ore e nell'intervallo $1-24$ ore

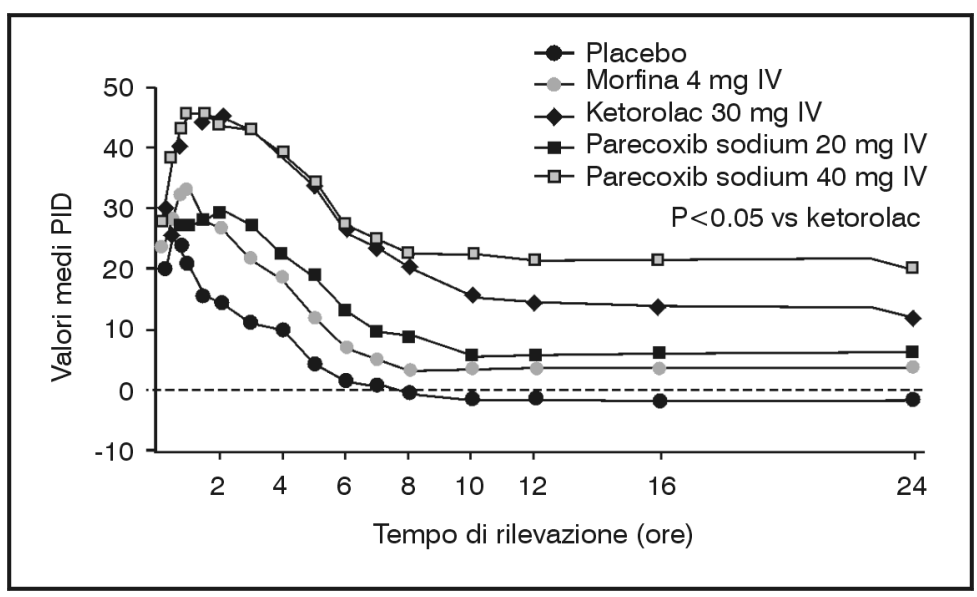

Figura 2

Valori di PID registrati ai diversi tempi di rilevazione

state randomizzate a ricevere una prima dose del trattamento (parecoxib $20 \mathrm{mg}$, parecoxib 40 $\mathrm{mg}$, ketorolac $30 \mathrm{mg}$, morfina $4 \mathrm{mg}$ o placebo, tutti endovena). La PID (Pain Intensity Difference) ottenuta con ketorolac e parecoxib è risultata significativamente superiore a quella degli altri gruppi per tutto il periodo compreso tra i 30 minuti e le 24 ore successive alla somministrazione. A partire dalla sesta ora dopo la somministrazione parecoxib $40 \mathrm{mg}$ è risultato superiore a ketorolac (figura 2).

Ventiquattro ore dopo, la terapia è proseguita con parecoxib 20 o $40 \mathrm{mg}$ o con ketorolac $30 \mathrm{mg}$ (fase a dosi multiple). L'efficacia di parecoxib, in entrambe le fasi, è risultata comparabile a quella di ketorolac, e, nella fase a dose unica è stata superiore a quella ottenuta con morfina o placebo.

Nello studio di Malan et al [15] l'efficacia analgesica di un'unica dose intramuscolo di $40 \mathrm{mg}$ di parecoxib è stata confrontata con quella ottenuta con una dose intramuscolo di 


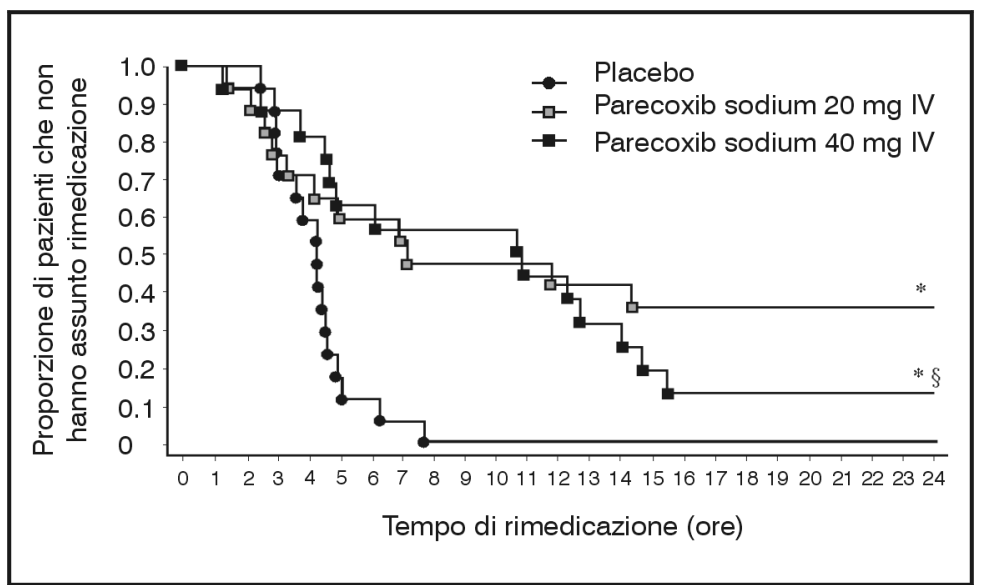

Figura 3

Curve di proporzione dei pazienti che non richiedono la rimedicazione negli intervalli di tempo valutati

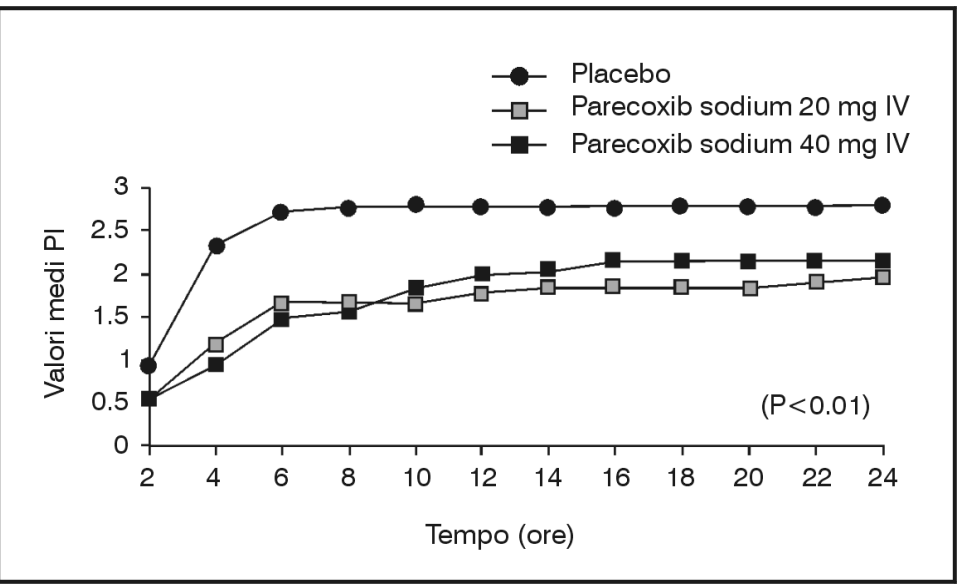

Figura 4

Valori medi di PI ai vari tempi di rilevazione

morfina $6 \mathrm{mg}$ o $12 \mathrm{mg}$ o placebo, in 264 donne sottoposte a laparatomia per un intervento ginecologico. L'efficacia analgesica del parecoxib è risultata paragonabile a quella della morfina a $12 \mathrm{mg}$ (ma significativamente maggiore rispetto a quella da morfina $6 \mathrm{mg}$ e placebo) per quanto riguarda la valutazione del sollievo totale dal dolore, superiore per quanto riguarda la durata dell'azione analgesica.

La riduzione complessiva del consumo di oppiodi grazie alla terapia concomitante con parecoxib, è stata valutata negli studi di Tang et al [16] e di Wender et al [17]. In entrambi gli studi la somministrazione di parecoxib (a dosi di 20 o $40 \mathrm{mg}$ ) è stata in grado di ridurre in modo significativo il consumo di oppioidi nella popolazione studiata.

L'efficacia antidolorifica di parecoxib somministrato prima dell'intervento chirurgico, è stata valutata in due ricerche. Nel primo studio [18], i partecipanti hanno ricevuto la dose del trattamento 30-45 minuti prima dell'inizio dell'operazione e sono stati seguiti per le 24 ore successive. I pazienti trattati con parecoxib 20 $\mathrm{mg}$ e $40 \mathrm{mg}$ hanno richiesto la rimedicazione dopo un tempo più lungo che quelli trattati con il placebo, a riprova dell'efficacia antalgica della somministrazione preoperatoria. La percentuale di pazienti che ha richiesto la rimedicazione è risultata significativamente più bassa nel gruppo parecoxib $20 \mathrm{mg}$ che in quello placebo (figura 3 ).

La percentuale di pazienti che ha terminato lo studio è stata significativamente maggiore nei gruppi sottoposti al trattamento attivo che in quello placebo. Inoltre, nell'intervallo di tempo compreso tra le quattro e le ventiquattro ore successive all'intervento, $i$ valori di PI registrati nei gruppi in trattamento con parecoxib sodico 20 e $40 \mathrm{mg}$ sono risultati significativamente inferiori $(\mathrm{p}<0,01)$ rispetto a quelli del gruppo placebo (figura 4). Anche nello studio di Desjardins et al [19] il tempo di rimedicazione è risultato superiore nei soggetti trattati preoperativamente con parecoxib che nei pazienti che avevano ricevuto placebo, ma le differenze non sono statisticamente significative.

In conclusione, sulla base dei trial clinici effettuati, parecoxib 20 o $40 \mathrm{mg} /$ die ha mostrato i seguenti risultati:

rapidità d'insorgenza dell' azione analgesica:

sovrapponibile a ketorolac 30-60 mg /die

superiore a ibuprofene $400 \mathrm{mg} /$ die e morfina $4 \mathrm{mg} / \mathrm{die}$

durata dell'effetto antalgico:

- sovrapponibile e/o superiore a ketorolac $30-60 \mathrm{mg} /$ die e superiore a morfina $4 \mathrm{mg} /$ die

\section{STUDI DI SICUREZZA}

La sicurezza del parecoxib nel trattamento del dolore successivo a chirurgia, può essere valutata prendendo in considerazione la tollerabilità osservata negli studi di efficacia appena descritti (tabella 3), specifici studi di sicurezza gastrointestinale e di impatto sulla funzionalità renale e piastrinica, nonché studi di interazione con alcuni dei farmaci più utilizzati in ambito chirurgico.

\section{Tollerabilità negli studi di efficacia}

Parecoxib ha evidenziato in ciascuno di essi una buona tollerabilità nel trattamento a breve termine del dolore post-operatorio. L'incidenza e la gravità degli effetti indesiderati nel complesso non differiscono da quelle che si verificano con il placebo. Non c'è evidenza di episodi di sanguinamento gastrointestinale o della ferita operatoria. Né la via di somministrazione 


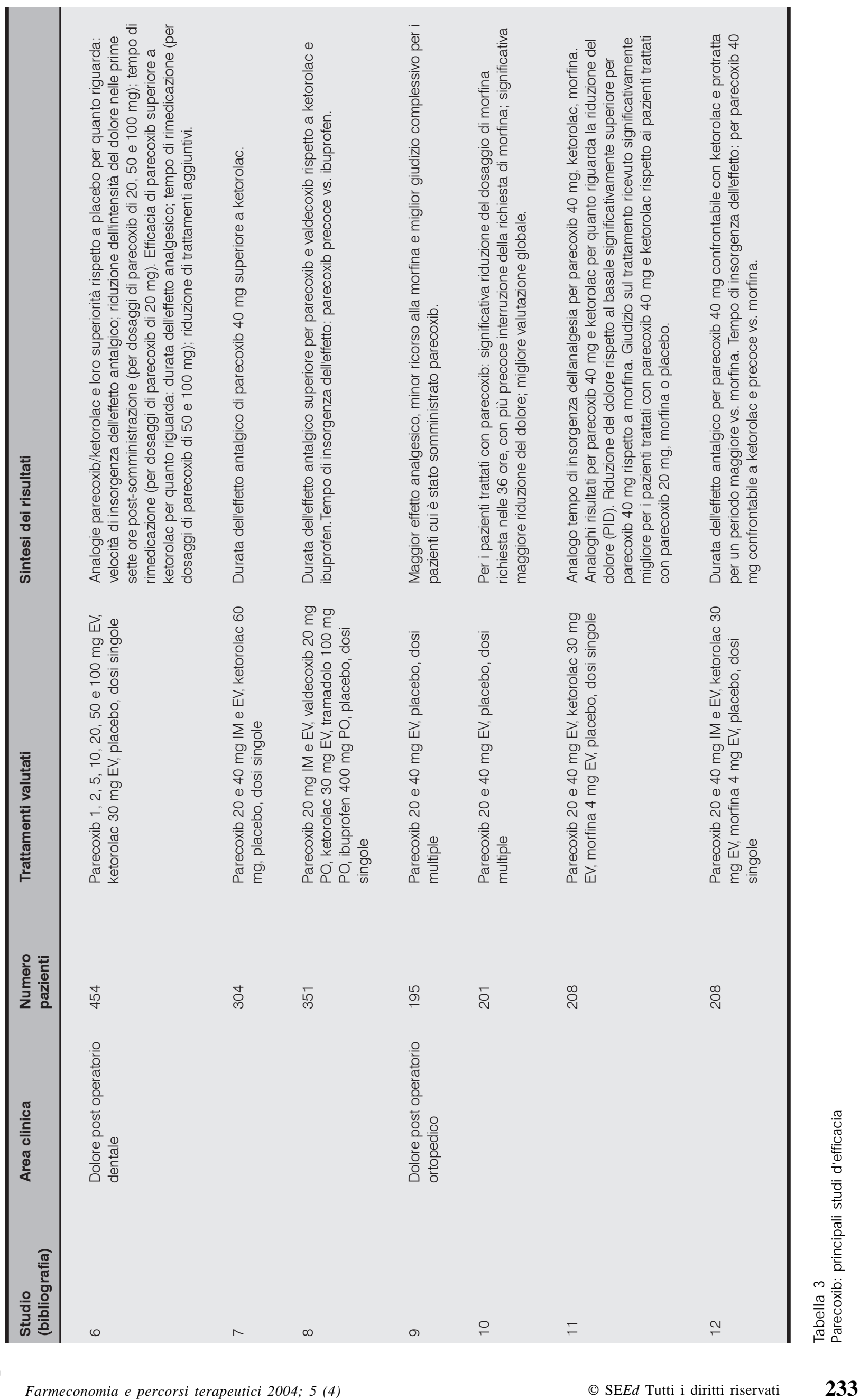




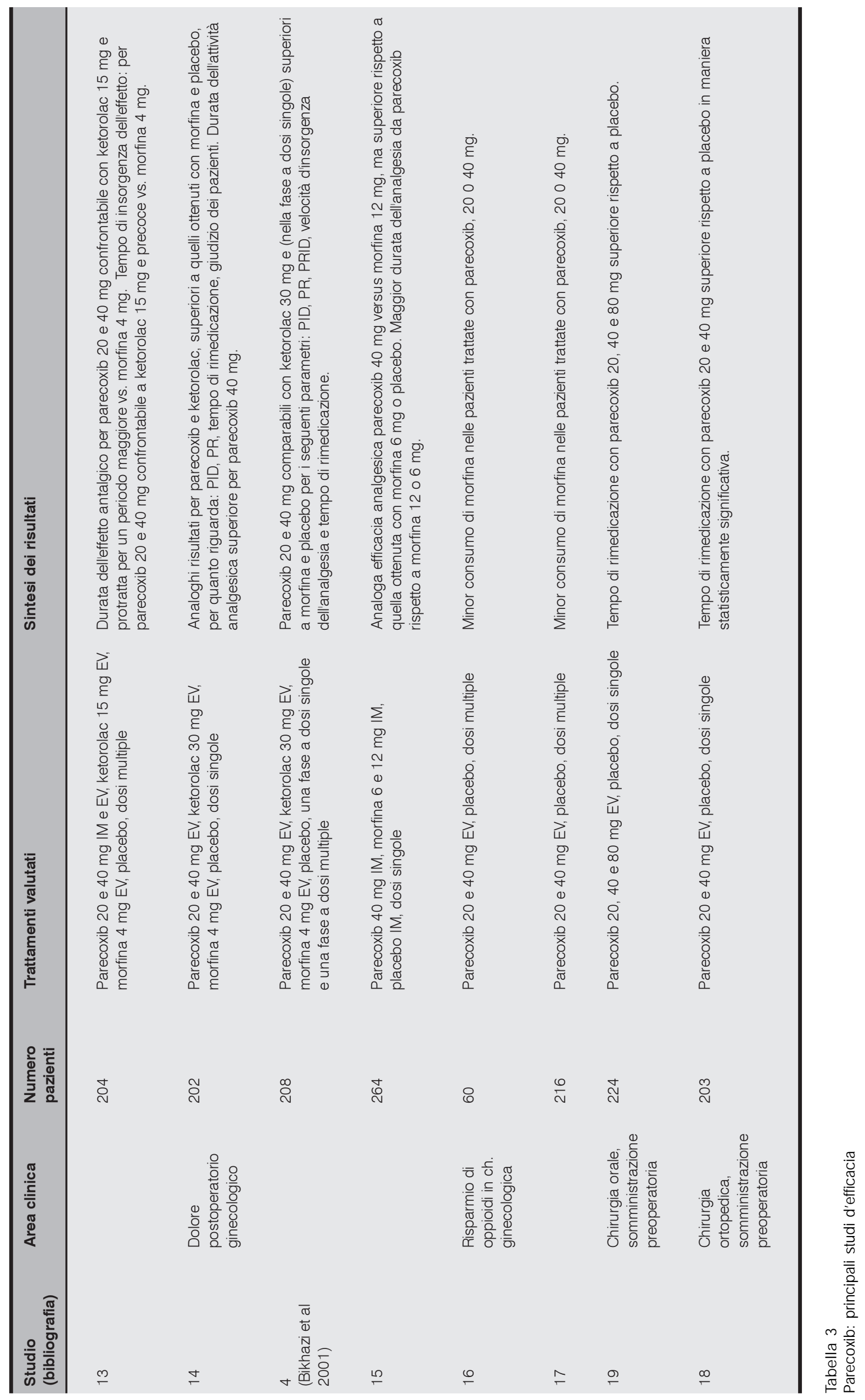




\begin{tabular}{|c|c|c|c|}
\hline $\begin{array}{l}\text { Studio } \\
\text { (bibliografia) }\end{array}$ & $\begin{array}{l}\text { Numero } \\
\text { pazienti }\end{array}$ & Trattamenti valutati & Risultati \\
\hline 20 & 166 & $\begin{array}{l}\text { Parecoxib } 20 \text { mg EV BID, naprossene } \\
500 \text { mg PO BID, ketorolac } 30 \text { mg EV } \\
\text { QID, placebo }\end{array}$ & $\begin{array}{l}\text { Percentuale di ulcere: } 0 \% \text { placebo, } 2 \% \text { parecoxib, } 39 \% \\
\text { ketorolac, } 17 \% \text { naprossene }\end{array}$ \\
\hline 21 & $\begin{array}{l}17 \\
\text { (anziani) }\end{array}$ & $\begin{array}{l}\text { Parecoxib } 10 \text { mg EV BID, naprossene } \\
500 \text { mg PO BID, ketorolac } 15 \text { mg EV } \\
\text { QID, placebo }\end{array}$ & $\begin{array}{l}\text { Percentuale di ulcere: } 40 \% \text { placebo, } 0 \% \text { parecoxib, } 100 \% \\
\text { ketorolac, } 50 \% \text { naprossene }\end{array}$ \\
\hline 22 & 123 & $\begin{array}{l}\text { Parecoxib } 40 \text { mg EV BID, ketorolac } \\
30 \text { mg EV QID, placebo }\end{array}$ & $\begin{array}{l}\text { Percentuale di ulcere: } 0 \% \text { placebo, } 0 \% \text { parecoxib, } 28 \% \\
\text { ketorolac. Percentuale di ulcere + erosioni: } 12 \% \text { placebo, } \\
7 \% \text { parecoxib, } 85 \% \text { ketorolac }\end{array}$ \\
\hline 23 & $\begin{array}{l}94 \\
\text { (anziani) }\end{array}$ & $\begin{array}{l}\text { Parecoxib } 40 \text { mg EV BID, ketorolac } \\
15 \text { mg EV QID, placebo }\end{array}$ & $\begin{array}{l}\text { Percentuale di ulcere: } 0 \% \text { placebo, } 0 \% \text { parecoxib, } 23 \% \\
\text { ketorolac. Percentuale di ulcere + erosioni: } 6 \% \text { placebo, } \\
21 \% \text { parecoxib, } 90 \% \text { ketorolac }\end{array}$ \\
\hline
\end{tabular}

Tabella 4

Studi endoscopici di tollerabilità gastrointestinale

(endovena o intramuscolo) né il dosaggio (20 o $40 \mathrm{mg}$ ) sono significativamente correlati con l'incidenza degli eventi avversi (figura 5). Inoltre, rispetto al placebo, in seguito a trattamento con parecoxib la febbre è risultata significativamente diminuita $[9,14] \mathrm{e}$, grazie alla riduzione dell'uso di morfina, anche l'incidenza di alcuni effetti indesiderati correlati all'uso degli analgesici oppioidi risulta ridotta [10].

Questi effetti possono favorire una migliore e quindi più rapida ripresa da parte del paziente dopo l'intervento.

\section{Sicurezza gastrointestinale}

Come evidenziato in tabella 4 , negli studi effettuati parecoxib dimostra una buona sicurezza/tollerabilità gastrointestinale sia nell'adulto che nell' anziano, pur essendo quest'ultimo normalmente più a rischio di emorragie gastrointestinali.

In sintesi, l'incidenza di ulcere gastro-intestinali osservate endoscopicamente dopo somministrazione endovenosa di parecoxib sodico, alle dosi di 10, 20 e 40 mg, BID, è risultata:

- simile a quella riscontrata con placebo

- inferiore, in maniera statisticamente significativa, a quella osservata con ketorolac 15 e $30 \mathrm{mg}$ e.v. QID

- inferiore, in maniera statisticamente significativa, a quella osservata con naprossene $500 \mathrm{mg}$ PO BID.

La figura 6 riassume le incidenze osservate sotto forma di grafico.

\section{Funzionalità piastrinica e renale}

Studi clinici controllati [24,25], condotti su volontari, in parte anziani, hanno valutato gli

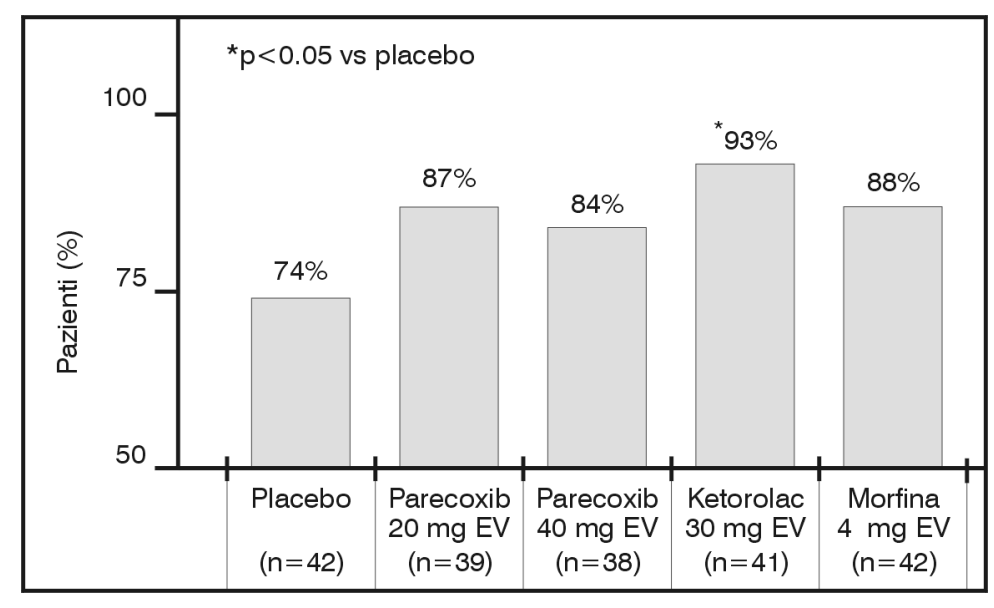

Figura 5

Incidenza di eventi avversi [14]

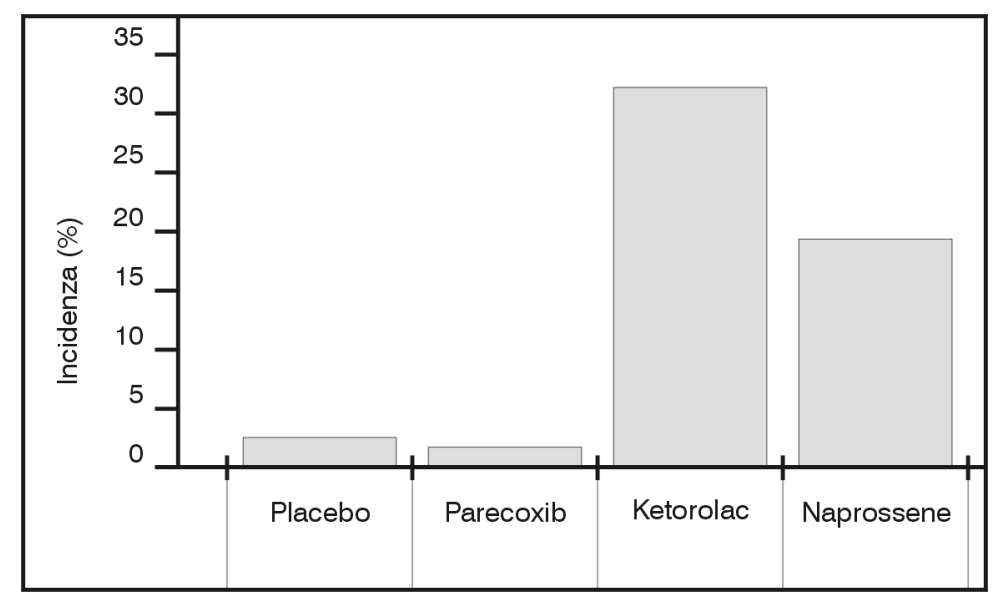

Figura 6

Incidenza (\%) di ulcere gastrointestinali

effetti della somministrazione di parecoxib sulla funzionalità piastrinica e renale. Grazie alla sua azione selettiva su COX-2, parecoxib, diversamente dai FANS non COX-2 selettivi, non modifica l'aggregazione piastrinica e i tempi di sanguinamento. Ciò rende il farmaco poten- 


\begin{tabular}{|c|c|c|c|}
\hline Studio (bibliografia) & $\begin{array}{l}\text { Numero } \\
\text { pazienti }\end{array}$ & Trattamenti valutati & Risultati \\
\hline 24 & 65 & $\begin{array}{l}\text { Parecoxib } 10 \text { o } 20 \text { mg EV BID, } \\
\text { ketorolac } 30 \mathrm{mg} \text { EV QID, } \\
\text { placebo }\end{array}$ & $\begin{array}{l}\text { Parecoxib non ha effetto sull'aggregazione piastrinica e } \\
\text { minor effetto di ketorolac sull'escrezione urinaria di sodio }\end{array}$ \\
\hline 25 & $\begin{array}{l}62 \\
\text { (anziani) }\end{array}$ & $\begin{array}{l}\text { Parecoxib } 10 \text { o } 40 \text { mg EV BID, } \\
\text { ketorolac } 15 \text { mg EV QID, } \\
\text { placebo }\end{array}$ & $\begin{array}{l}\text { Parecoxib non ha effetto sull'aggregazione piastrinica; } \\
\text { effetti urinari sulle PG e sull'escrezione di sodio } \\
\text { confrontabili a ketorolac }\end{array}$ \\
\hline 25 & 48 & $\begin{array}{l}\text { Parecoxib } 10 \text { o } 40 \text { mg EV BID, } \\
\text { ketorolac } 15 \mathrm{mg} \text { EV QID, } \\
\text { placebo }\end{array}$ & $\begin{array}{l}\text { Parecoxib non ha effetto sull'aggregazione piastrinica; } \\
\text { effetti urinari sulle PG e sull'escrezione di sodio } \\
\text { confrontabili a ketorolac }\end{array}$ \\
\hline $\begin{array}{l}4 \\
\text { (Katz et al, 2003) }\end{array}$ & 1704 & $\begin{array}{l}\text { Parecoxib } 20 \text { o } 40 \text { mg EV BID, } \\
\text { placebo }\end{array}$ & $\begin{array}{l}\text { Rispettivamente per parecoxib } 20 \text { mg, parecoxib } 40 \text { mg e } \\
\text { placebo, l'incidenza dei sanguinamenti è risultata pari a: } \\
5,8 \%, 1,5 \%, 2,6 \% \text { per somministrazione pre-operatoria; } \\
6,2 \%, 7,7 \%, 5,1 \% \text { per somministrazione post-operatoria; } \\
6,3 \%, 6,0 \%, 5,3 \% \text { per somministrazione post-operatoria } \\
\text { per più di } 1 \text { giorno }\end{array}$ \\
\hline
\end{tabular}

Tabella 5

Studi sulla funzionalità piastrinica e renale

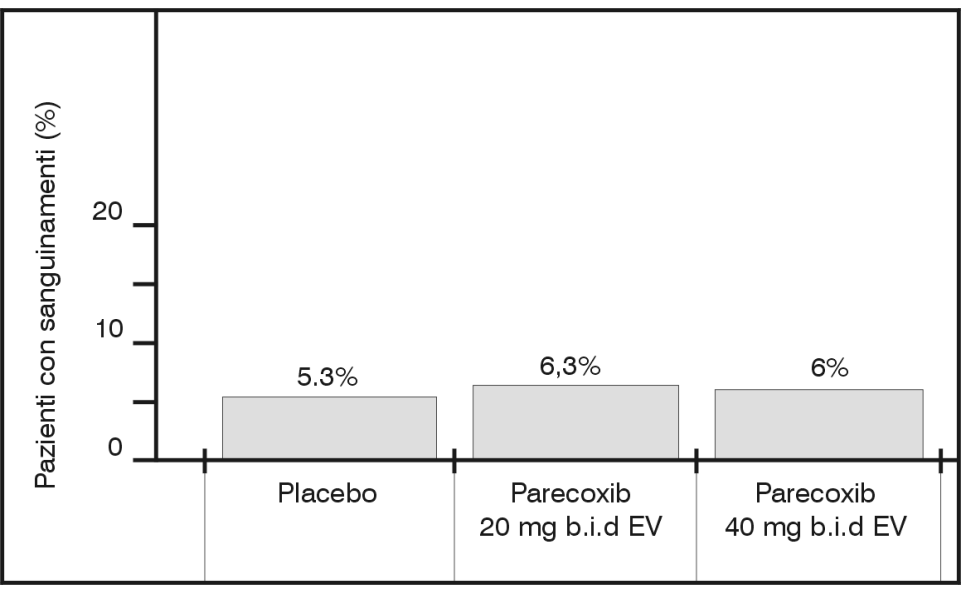

Figura 7

Parecoxib mostra un'incidenza di eventi avversi di natura emorragica paragonabile al placebo [4] (Katz et al, 2003)

zialmente più sicuro per esempio del ketorolac, essendo associato ad un minor rischio di sanguinamento post-operatorio. I principali risultati sono riportati in tabella 5. Nello studio di Katz et al [4], è stata indagata l'incidenza dei sanguinamenti in seguito alla somministrazione endovena di parecoxib $20 \mathrm{mg}$, parecoxib $40 \mathrm{mg}$ o placebo (figura 7).
Nello stesso studio, anche l'incidenza di complicanze correlate alla guarigione delle ferite, di eventi renali e di eventi cardiovascolari è risultata paragonabile al placebo.

In pazienti in terapia con acido acetilsalicilico, inoltre, non sono state riscontrate interazioni clinicamente significative con parecoxib $40 \mathrm{mg}$ [26].

\section{Interazioni farmacologiche con altri farmaci}

Nell'ambito del piano di sviluppo clinico del farmaco, sono stati condotti specifici studi di interazione farmacocinetica e/o farmacodinamica con altri farmaci di frequente utilizzo nel suo campo di applicazione. I principali risultati di questi studi, con eccezione di quello già ricordato che valutava l'influenza sull'aggregazione piastrinica in pazienti in terapia con ASA, sono riportati in tabella 6.

La mancanza di interazioni significative con fentanil, alfentanil, midazolam e propofol risulta piuttosto importante, vista la frequenza con cui tali farmaci vengono impiegati nei pazienti chirurgici.

\begin{tabular}{ll}
\hline Studio di interazione (bibliografia) & Sintesi del risultato \\
\hline Parecoxib e eparina [27] & Non sono state rilevate interazioni clinicamente significative \\
Parecoxib 40 mg BID e propofol [28] & Non sono state rilevate interazioni clinicamente significative \\
Parecoxib 40 mg BID e midazolam EV [29] & Non sono state rilevate interazioni clinicamente significative \\
Parecoxib 40 mg BID e fentanil/alfentanil [30] & Non sono state rilevate interazioni clinicamente significative \\
Parecoxib 10 mg BID e warfarin [31] & Interazione significativa con R-warfarin, non con S-warfarin \\
\hline
\end{tabular}

Tabella 6

Studi di interazione con altri farmaci 


\section{CONSIDERAZIONI FARMACOECONOMICHE}

\section{Definizione del problema}

In Italia ancora oggi interventi chirurgici che potrebbero essere effettuati in regime di day-surgery vengono spesso effettuati con l'ospedalizzazione del paziente. Secondo i dati forniti dal Ministero della Salute, nel 1999, su circa 4 milioni di interventi chirurgici eseguiti in Italia, la percentuale di quelli eseguiti in regime di day-surgery è stata del $14,5 \%$, contro il $60 \%$ degli USA, il 30\% del Regno Unito, il $20 \%$ della Francia (figura 8) [32].

Il dolore post-chirurgico è uno dei fattori che può rendere conto del sotto-utilizzo del regime di day-surgery: nelle $24-48$ ore successive ad un intervento di chirurgia minore eseguibile in day-surgery o in artroscopia, oltre il $40 \%$ dei pazienti accusa dolore di grado moderato/severo $[33,34]$.

I diversi protocolli di trattamento farmacologico del dolore post-chirurgico, sono generalmente basati sull'uso di analgesici periferici appartenenti alla classe dei FANS e/o di analgesici oppioidi. In particolare la somministrazione di un FANS per via parenterale nel post-operatorio è sufficiente, nella maggior parte dei casi, a garantire un adeguato livello di analgesia post-operatoria ai pazienti sottoposti ad interventi chirurgici o a procedure diagnostiche dolorose eseguibili in day surgery $\mathrm{o}$ in ambulatorio. Un elenco non esaustivo degli interventi di chirurgia minore e delle procedure diagnostiche dolorose che richiedono un trattamento analgesico con un FANS per via parenterale è riportato nella tabella 7 .

Come scritto nell' introduzione del presente articolo, questa classe di farmaci mostra però una elevata incidenza di effetti collaterali.

Per esempio il ketorolac, che è uno dei farmaci più utilizzati nel trattamento del dolore post-chirurgico, accanto ad una elevata efficacia analgesica, presenta un alto rischio di reazioni avverse a carico della mucosa gastrointestinale e della funzione piastrinica,

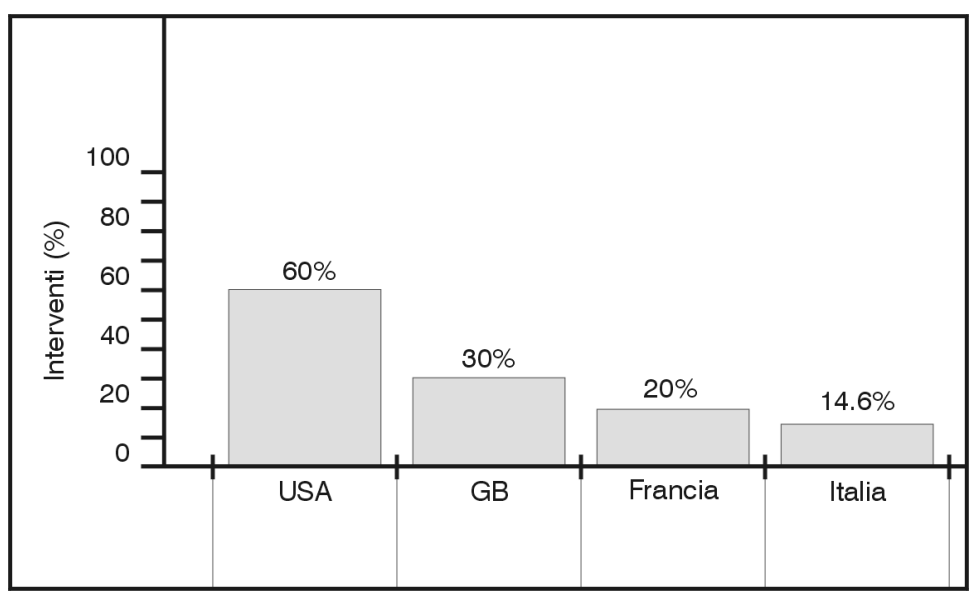

Figura 8

Percentuale di interventi in regime di day surgery sul totale degli interventi chirurgici in vari paesi

tanto che il Ministero della Salute ha fatto inserire avvertenze e limitazioni nel foglio illustrativo del prodotto.

Numerosi studi confermano il problema. Rispetto ai pazienti trattati con gli altri FANS, per i pazienti trattati con ketorolac, risultano superiori sia il rischio relativo di sviluppare lesioni peptiche $(R R=4,2)$ [35], sia il rischio di essere ricoverati per ulcera gastroduodenale (rischio ben 3,4 volte superiore) [36].

In base ai calcoli effettuati nello studio di Chen et al [37], condotto su un campione di 1,5 milioni di dimissioni ospedaliere, l'impatto economico delle reazioni avverse al ketorolac può comportare costi di ospedalizzazione superiori del $20 \%$ rispetto al costo medio dei pazienti trattati con gli altri FANS.

Parecoxib, grazie alla sua intensa e prolungata azione analgesica, al suo eccellente profilo di sicurezza e alla sua disponibilità in formulazione per uso endovenoso ed intramuscolare, può costituire una valida alternativa, consentendo significativi risparmi sui costi associati alle reazioni avverse ai FANS e sul costo dell'assistenza ospedaliera ed extra-ospedaliera dei pazienti che devono essere sottoposti ad interventi chirurgici eseguibili in day-surgery o che devono affrontare procedure diagnostiche dolorose.
Interventi chirurgici eseguibili in ambulatorio/ day hospital

Ernia inguinale - artroscopia - varicectomia decompressione tunnel carpale - cataratta/glaucoma estrazioni dentarie - emorroidectomia - laparoscopia ginecologica - varicocele - tonsillectomia colecistectomia laparoscopica - miringotomia
Procedure diagnostiche dolorose

Biopsie (escissionali, incisionali, ad ago aspirato) - prelievo e analisi liquidi biologici (liquido pleurico, sinoviale, cerebrospinale, pericardico, peritoneale) - laringoscopia - cistoureteroscopia - caterizzazioni (uretrali, cardiache) - puntura lombare- aspirazione del midollo osseo

Tabella 7

Interventi e procedure che richiedono un trattamento antidolorifico 


\section{Metodo di valutazione economica}

La ditta produttrice ha presentato al Ministero della Salute italiano alcune valutazioni circa il possibile impatto economico sul SSN conseguente all'introduzione del parecoxib, in alternativa al ketorolac, nel trattamento parenterale del dolore post-chirurgico dei pazienti sottoposti ad interventi di chirurgia minore gestibili in day surgery. La valutazione farmacoeconomica è stata attuata secondo la tecnica "minimizzazione dei costi". È stata assunta l'ipotesi conservativa che non vi siano differenze significative tra l'efficacia analgesica dei due farmaci confrontati, così come è in effetti emerso da alcuni trials clinici, che hanno dimostrato come la dose parenterale di parecoxib di $40 \mathrm{mg} /$ die abbia un'efficacia analgesica almeno uguale a quella parenterale di ketorolac di $30 \mathrm{mg} / \mathrm{die}$.

Sono stati stimati e confrontati i costi totali diretti del trattamento analgesico parenterale di parecoxib o di ketorolac, cioè sia il costo di acquisto del farmaco sia il costo delle reazioni avverse associate al loro uso. Inoltre è stato stimato il potenziale risparmio derivante dalla trasformazione di una certa percentuale di ricoveri ordinari in ricoveri brevi tipo day-surgey, conseguente ad una più sicura ed efficiente gestione del dolore acuto postoperatorio resa possibile dall'introduzione in terapia del parecoxib. Attualmente, infatti, la gestione del dolore post-chirurgico nei pazienti sottoposti ad interventi di chirurgia sia maggiore sia minore è ancora spesso inadeguata [25], con conseguenze tutt'altro che trascurabili per il benessere del paziente e per i costi evitabili caricati sull'ospedale e soprattutto sul SSN (ospedalizzazioni prolungate, risorse aggiunte per la gestione degli effetti indesiderati, ricoveri ordinari piuttosto che in day hospital).

La migliore maneggevolezza d'uso e la minore tossicità del parecoxib, rispetto al ketorolac, può consentire significativi risparmi nell'utilizzo delle risorse sanitarie a livello ospedaliero (per esempio riduzione di giorni di degenza e di costi di trattamento) e, più in generale, a livello del SSN.

\section{Costi d'acquisto}

Poiché per entrambi i farmaci è necessario lo stesso materiale per la loro somministrazione endovena o intramuscolare, si considerano qui solo i costi del farmaco. Il prezzo al pubblico del ketorolac iniettabile è di 1,50 euro per una fiala da $30 \mathrm{mg}$. Calcolando lo sconto obbligatorio del $50 \%$ per le forniture ospedaliere, il prezzo di un giorno di terapia parenterale con $30 \mathrm{mg}$ di ketorolac risulta di 0,75 euro. Il prezzo al pubblico del parecoxib iniettabile è di 14,61 euro per una fiala da $40 \mathrm{mg}$. Calcolando lo sconto obbligatorio del $50 \%$ per le forniture ospedaliere, il prezzo di un giorno di terapia parenterale con $40 \mathrm{mg}$ di parecoxib risulta di 7,30 euro. Un giorno di terapia con parecoxib, dunque, costa all'ospedale 6,55 euro in più di un giorno di terapia con ketorolac.

In caso di prosecuzione della terapia antalgica al domicilio del paziente, opportunità resa possibile dalla somministrazione intramuscolare e dalla tollerabilità del parecoxib, i costi di acquisto (14,61 euro/die) graverebbero sul paziente, in quanto il farmaco è attualmente a totale carico dell'assistito (fascia C).

\section{Costi per la gestione degli effetti indesiderati}

I costi associati agli effetti avversi del ketorolac sono stati stimati utilizzando i dati di costo di una survey [38] effettuata su un database della Regione Friuli Venezia Giulia. Dal database, che contiene i dati relativi alle prescrizioni, alle ospedalizzazioni, agli esami diagnostici e ai morti relativi ad una popolazione formata da 1,2 milioni di residenti, è stata estratta una coorte di pazienti che avessero ricevuto almeno una prescrizione di FANS. Di questa coorte è stato valutato il numero di interventi medici per disturbi del tratto gastrointestinale superiore conseguenti a terapia con FANS e i relativi costi, attribuiti sulla base della loro sovrapposizione temporale con l'esposizione al FANS.

Il costo medio giornaliero, limitatamente agli eventi gastrointestinali, per paziente trattato con ketorolac è risultato di 1,73 euro, mentre per i pazienti che hanno ricevuto altri FANS iniettivi è stato di 0,91 euro.

Il risparmio giornaliero sugli eventi avversi, conseguente alla miglior tollerabilitá del parecoxib, potrebbe quindi oscillare da un minimo di 0,82 (1,73-0,91) euro, se assumiamo che il costo delle reazioni avverse al parecoxib sia sovrapponibile a quello della media dei FANS tradizionali, a un massimo di 1,73 euro, se assumiamo che il farmaco sia privo di reazioni avverse gravi responsabili di indurre significativi aumenti dei costi sanitari.

Per stimare il costo degli effetti avversi per paziente o per operazione chirurgica, tale risparmio deve essere moltiplicato per il numero di giorni in cui i costi derivati dalle reazioni avverse stesse e dall'ospedalizzazione del paziente gravano sul SSN, numero di giorni presumibilmente variabile a seconda del tipo di intervento per cui è richiesta l'analgesia. 


\section{Stima dei risparmi potenziali conseguenti alla miglior gestione del dolore post-operatorio}

L'adeguato trattamento del dolore postoperatorio con parecoxib, da iniziare in ospedale e continuare a domicilio, potrebbe consentire la gestione di alcuni pazienti in day hospital anziché in regime di ricovero ordinario. Il potenziale risparmio derivante da questo tipo di gestione del paziente è stato calcolato in base ai dati del Ministero della Salute riguardanti le ospedalizzazioni dell' anno 1999.

Sono stati presi in considerazione $\mathrm{i}$ dati di numerosità, tipologia di ricovero e costi totali associati ai DRG che compaiono nell'elenco ministeriale dei DRG "a rischio di inappropriatezza" e per i quali l'efficacia e la tollerabilità di parecoxib potrebbero permettere la gestione di alcuni pazienti in regime di day hospital. In tabella 8 sono riportati i dati relativi a cinque di questi DRG.

L'eventuale risparmio per il SSN derivante dallo switch dalla degenza ordinaria al day hospital è stato calcolato come segue:

Esempio:

DRG 187 -Estrazioni e riparazioni dentali

Il rimborso per la degenza ordinaria è di 1656,28 euro, contro 371,33 euro per il DH, la differenza risulta quindi di 1284,95 euro.

Considerando che nel 199916.058 pazienti sono stati sottoposti ad intervento in regime di ricovero ordinario, se anche solo l'1\% (161) di essi venisse trattato in regime di DH e proseguisse la terapia a domicilio grazie alla tera- pia con parecoxib, il potenziale risparmio per il SSN sarebbe di $161 * 1.284,95=206.343$ euro/ anno.

La metodologia di calcolo sopra esemplificata è stata applicata ai 7 DRG che presentano caratteristiche compatibili con l'approccio in day hospital (estr. e rip. dentale, interventi sul ginocchio, artroscopia, affezioni mediche del dorso, dilatazione e raschiamenti, conizzazione).

In base ai calcoli effettuati, è stato stimato che lo switch dal regime di ricovero ordinario alla gestione in DH dell' $1 \%(\mathrm{n} .=715)$ dei pazienti trattati con parecoxib porterebbe ad un risparmio totale per il SSN di 723.320 euro nel primo anno, che suddiviso sul totale della popolazione da trattare $(\mathrm{n}=71.500)$, comporterebbe un risparmio di 10,12 euro per paziente trattato.

\section{CONCLUSIONI}

Parecoxib sodico è il primo inibitore selettivo della COX-2 disponibile per la somministrazione parenterale. La sua possibile somministrazione per via endovenosa o intramuscolare ne permette l'utilizzo nel trattamento del dolore acuto post-operatorio, per la gestione del quale attualmente sono utilizzati i FANS tradizionali, in particolar modo il ketorolac, il cui beneficio terapeutico è ridotto dalla frequente induzione di effetti collaterali sfavorevoli a carico del tratto gastroenterico. Studi clinici hanno dimostrato che dosi di 20$40 \mathrm{mg} /$ die di parecoxib hanno un'efficacia almeno paragonabile a ketorolac (30-60 $\mathrm{mg} / \mathrm{die})$, senza evidenza di eventi avversi a carico della

\begin{tabular}{|c|c|c|c|c|}
\hline DRG & $\begin{array}{l}\text { N. ricoveri } \\
\text { ordinari } \\
\text { (\% sul totale) }\end{array}$ & $\begin{array}{l}\text { N. ricoveri in } \mathrm{DH} \\
\text { (\% sul totale) }\end{array}$ & $\begin{array}{l}\text { Costo totale ricoveri } \\
\text { ordinari } \\
\text { (\% sul totale) } \\
\text { in euro }\end{array}$ & $\begin{array}{l}\text { Costo totale } \\
\text { ricoveri in } \mathrm{DH} \\
\text { (\% sul totale) } \\
\text { in euro }\end{array}$ \\
\hline $\begin{array}{l}160 \\
\text { (Intervento per ernie, eccetto } \\
\text { inguinale e femorale, non } \\
\text { complicate) }\end{array}$ & $\begin{array}{l}21.141 \\
(90,7)\end{array}$ & $\begin{array}{l}2.163 \\
(9,3)\end{array}$ & $\begin{array}{l}50.541 .365 \\
(96,3)\end{array}$ & $\begin{array}{l}1.941 .508 \\
(3,7)\end{array}$ \\
\hline $\begin{array}{l}187 \\
\text { (Estrazioni e riparazioni dentali) }\end{array}$ & $\begin{array}{l}16.059 \\
(57,3)\end{array}$ & $\begin{array}{l}11.981 \\
(42,7)\end{array}$ & $\begin{array}{l}26.598 .200 \\
(85,7)\end{array}$ & $\begin{array}{l}4.448 .904 \\
(14,3)\end{array}$ \\
\hline $\begin{array}{l}222 \\
\text { (Intervento sul ginocchio in } \\
\text { artroscopia, non complicato) }\end{array}$ & $\begin{array}{l}108.510 \\
(87,5)\end{array}$ & $\begin{array}{l}15.566 \\
(12,5)\end{array}$ & $\begin{array}{l}298.641 .222 \\
(92,8)\end{array}$ & $\begin{array}{l}23.000 .010 \\
(7,2)\end{array}$ \\
\hline $\begin{array}{l}232 \\
\text { (Artroscopia) }\end{array}$ & $\begin{array}{l}29.420 \\
(86,4)\end{array}$ & $\begin{array}{l}4.646 \\
(13,6)\end{array}$ & $\begin{array}{l}58.132 .743 \\
(92,1)\end{array}$ & $\begin{array}{l}5.017 .261 \\
(7,9)\end{array}$ \\
\hline $\begin{array}{l}364 \\
\text { (Dilatazione e raschiamento, } \\
\text { no neoplasie maligne) }\end{array}$ & $\begin{array}{l}70.167 \\
(62,4)\end{array}$ & $\begin{array}{l}42.330 \\
(37,6)\end{array}$ & $103.134 .263 \quad(76,5)$ & $\begin{array}{l}31.633 .632 \\
(23,5)\end{array}$ \\
\hline
\end{tabular}

Tabella 8

Numerosità, tipologia di ricovero e costi totali di DRG a rischio di inappropriatezza 
mucosa del tratto gastroenterico superiore o delle piastrine. Si tratta pertanto di un farmaco maneggevole, efficace e sicuro, il che consente di immaginare un suo utilizzo anche per interventi ambulatoriali.

Sulla base di questi dati clinici, abbiamo sviluppato alcune considerazioni di ordine farmacoeconomico. Grazie all' assenza di effetti collaterali gastrointestinali, che causano un prolungamento dell'ospedalizzazione per molti pazienti trattati con i FANS, l'uso di parecoxib po- trebbe consentire una riduzione della durata media delle degenze nei reparti di chirurgia, risultando economicamente vantaggioso per l'ospedale, nonostante il maggior prezzo di acquisto.

Inoltre, la tollerabilità e la sicurezza che caratterizzano l'uso del parecoxib, permetterebbero di eseguire in regime di day hospital alcuni tipi di interventi chirurgici, con un conseguente doppio vantaggio economico per il SSN: una riduzione del rimborso e un aumento dell'efficienza ospedaliera.

\section{BIBLIOGRAFIA}

1. McMurray RW and Hardy KJ. COX-2 inhibitors: today and tomorrow. Am J Med Sci 2002;323(4):181-9.

2. Smith CJ, Zhang Y, Koboldt CM, Muhammad J, Zweifel BS, Shaffer A, Talley JJ, Masferrer JL, Seibert K, Isakson PC. Pharmacological analysis of cyclooxygenase-1 in inflammation. Proc Natl Acad Sci U S A. 1998 Oct 27;95(22):13313-8

3. Samad TA, Moore KA, Sapirstein A, Billet S, Allchorne A, Poole S, Bonventre JV, Woolf CJ Interleukin-1betamediated induction of Cox-2 in the CNS contributes to inflammatory pain hypersensitivity. Nature. 2001 Mar 22;410(6827):471-5.

4. Parecoxib sodico EV/IM. Acta Pharmacologica 1 anno VIII. J Medical Books Edizioni S.r.l.

5. Dynastat. Riassunto delle Caratteristiche di Prodotto.

6. Mehlisch DR, Desjardins PJ, Daniels S, et al. Single doses of parecoxib sodium intravenously are as effective as ketorolac in reducing pain after oral surgery. J Oral Maxillofac Surg 2003;61(9):1030-7.

7. Daniels SE, Grossman EH, Kuss ME, Talwalker S, Hubbard RC. A double-blind, randomized comparison of intramuscularly and intravenously administered parecoxib sodium versus ketorolac and placebo in a post-oral surgery pain model. Clin Ther. $2001 \mathrm{Jul} ; 23(7): 1018-31$.

8. Integrated clinical and statistical report for a double-blind, placebo controlled, comparison of the analgesic activity of SC-69124A $20 \mathrm{mg} I M$, SC-69124A $20 \mathrm{mg}$ IV, SC-65872 $20 \mathrm{mg}$ PO, ketorolac $30 \mathrm{mg}$ IV, tramadol $100 \mathrm{mg}$ PO, ibuprofen $400 \mathrm{mg}$ PO and placebo in a postsurgical dental pain model. G.D. Searle \& Co., Data on file.

9. Hubbard RC, Naumann TM, Traylor L, et al. Parecoxib sodium has opioid-sparing effects in patients undergoing total knee arthroplasty under spinal anaesthesia. Br J Anaesth 2003;90(2):166-72. 
10. Malan TP Jr, Marsch g, Hakki SI, et al. Parecoxib sodium, a parenteral cyclooxygenase 2 selective inhibitor, improves morphine analgesia and is opioid-sparing following total hip arthroplasty. Anesthesiology 2003;98(4):950-6.

11. Rasmussen GL, Steckner K, Hogue G, et al. Intravenous parecoxib sodium for acute pain after orthopedic knee surgery. Am J Orthop 2002;31(6):336-43.

12. Final report for a single dose, double-blind, placebo controlled comparison of the analgesic activity of intravenously administered SC-69124A $20 \mathrm{mg}$, SC-69124A $40 \mathrm{mg}$, ketorolac $30 \mathrm{mg}$, morphine $4 \mathrm{mg}$, and placebo in post orthopedic surgery patients. G.D. Searle \& Co., Data on file.

13. Final report for a double-blind, placebo controlled comparison of the analgesic activity of SC-69124A $20 \mathrm{mg} I V$, SC-69124A $40 \mathrm{mg}$ IV, ketorolac $15 \mathrm{mg}$ IV, morphine $4 \mathrm{mg}$ IV, and placebo in post total hip replacement surgical patients. G.D. Searle \& Co., Data on file.

14. Barton Sf, Langeland FF, Snabes SC, et al. Efficacy and safety of intravenous parecoxib sodium in relieving acute postoperative pain following gynecologic laparotomy surgery. Anesthesiology 2002;97(2):306-14.

15. Malan TP, Gordon S, Traylor L, et al. Parecoxib sodium 40mg i.m. is as effective as morphine $12 \mathrm{mg}$ i.m. following gynecologic laparotomy. Eur J Anaesthesiol 2003;20 (suppl 30): A725. Poster.

16. Tang J, Li S, White PF, et al. Effect of parecoxib, a novel intravenous cyclooxygenase type-2 inhibitor, on the postoperative opioid requirenent and quality of pain control. Anesthesiology 2002;96(6):1305-9.

17. Wender RH et al. Parecoxib sodium demonstrates opioid sparing effects in post-laparotomy surgical patients. Fertil Steril 2001; 76(3, Suppl 3): S218-9.

18. Final report for a double-blind, placebo controlled evaluation of the analgesic activity of parecoxib sodium 20 mg IV and parecoxib sodium $40 \mathrm{mg}$ IV administered pre-operatively in a postsurgical orthopedic pain model. G.D. Searle \& Co., Data on file.

19. Desjardins $\mathrm{Pj}$ et al. The injectable cyclooxygenase-2-specific inhibitor parecoxib sodium has analgesic efficacy when administered preoperatively. Anesth Analg. 2001 Sep;93(3):721-7.

20. Revised report for a placebo controlled study, to compare the effects of intravenous administration of SC-69124A $20 \mathrm{mg}$ BID and ketorolac $30 \mathrm{mg}$ QID with orally administered naproxen $500 \mathrm{mg}$ BID on the upper gastrointestinal mucosa in healthy subjects. G.D. Searle \& Co., Data on file.

21. Harris SI et al. Upper gastrointestinal safety evaluation of parecoxib sodium, a new parenteral cyclooxygenase-2specific inhibitor, compared with ketorolac, naproxen, and placebo. Clin Ther. 2001 Sep;23(9):1422-8.

22. Harris SI et al. Parecoxib sodium, an injectable COX-2-specific inhibitor, showes improved upper gastrointestinal safety compared with ketorolac. Ann Emerg Med 2001;38(4,Suppl):S37.

23. Stolz RR et al. Upper GI mucosal effects of parecoxib sodium in healthy elderly subjects. Am J Gastroenterol 2002 JAn;97(1):65-71.

24. RCP autorizzato: SC-69124A $20 \mathrm{mg}$ BID and ketorolac $30 \mathrm{mg}$ QID with orally administered naproxen $500 \mathrm{mg}$ BID on the upper gastrointestinal mucosa in healthy subjects. G.D. Searle \& Co., Data on file.

25. Visentin M, Trentin L, de Marco R, Zanolin E. Knowledge and attitudes of Italian medical staff towards the approach and treatment of patients in pain. J Pain Symptom Manage.2001 Nov;22(5):925-30.

26. Integatred clinical and statistical report for a double-blind, placebo controlled comparison of the analgesic activity of SC-69124A $20 \mathrm{mg}$ IM, SC69124A $20 \mathrm{mg}$ IV, SC-65872 $20 \mathrm{mg}$ PO, ketorolac $30 \mathrm{mg}$ IV, tramadol $100 \mathrm{mg}$ PO, ibuprofen $400 \mathrm{mg}$ PO and placebo in postsurgical dental pain model. G.D. Searle \& Co., Data on file.

27. Investigator brochure for parecoxib sodium, a new parenteral inhibitor of cyclooxygenase-2 Forth edition, March 2001.

28. Ibrahim A, Park S, Feldman J, Karim A, Kharasch ED. Effects of parecoxib, a parenteral COX-2-specific inhibitor, on the pharmacokinetics and pharmacodynamics of propofol. Anesthesiology. 2002 Jan;96(1):88-95.

29. Ibrahim A, Karim A, Feldman J, , Kharasch E. The influence of parecoxib, a parenteral cyclooxyigenase-2-specific inhibitor, on the pharmacokinetics and clinical effects of midazolam. Anesth Analg. 2002 Sep;95(3):667-73.

30. Revised report for a double-blind,randomized, balanced cross-over, placebo controlled study to assess the effect of parecoxib sodium on the pharmacokinetics and pharmacodynamics of alfentanil and fentanyl. G.D. Searle \& Co., Data on file.

31. Karim A and Bradford DJQ. The COX-2-specific inhibitor parecoxib sodium does not affect warfarin pharmacokinetic and harmacodynamic parameters. Ann Emerg Med 2001; 38(4,Suppl):S37. 
Parecoxib nel trattamento del dolore post-operatorio: considerazioni farmacoeconomiche

32. Wetchler BV. Outpatient anesthesia. In: Barash PJ ed $2^{\text {nd }}$ ed. Philadelphia JB Lippincott 1992: 1389-1416.

33. De Lathouwer C, Poullier JP. How much ambulatory surgery in the world in 1996-1997 and trends? Ambul. Surg. 2000 Oct 1;8(4):191-210.

34. Svensson I, Sjostrom B, Haljamae H. Assessment of pain experiences after elective surgery. J Pain Symptom Manage. 2000 Sep;20(3):193-201.

35. Traversa G et al. Gastroduodenal toxicity of different nonsteroidal antiinflammatory drugs. Epidemiology. 1995 Jan;6(1):49-54.

36. Menniti-Ippolito $\mathrm{F}$ et al. Ketorolac use in outpatients and gastrointestinal hospitalization: a comparison with other non-steroidal anti-inflammatory drugs in Italy. Eur J Clin Pharmacol. 1998 Jul;54(5):393-7.

37. Chen $\mathrm{C}$ et al. Characteristics of parenteral ketorolac in the surgical population. Abstract presented at the mee-ting of the American Pain Society, 2000.

38. Sturkenboom MC et al. The iatrogenic costs of NSAID therapy: a population study. Arthritis Rheum. 2002 Apr15;47(2):132 